\title{
Catalog of Positions and Spectroscopic Properties of Galaxies in the A1367 Cluster
}

\author{
N. M. Lipovka ${ }^{1}$, J. C. Saucedo Morales ${ }^{2}$, A. A. Lipovka ${ }^{2}$, \\ A. V. Boldycheva ${ }^{3}$, and K. L. Maslennikov ${ }^{3}$
}

${ }^{1}$ Special Astrophysical Observatory, RAS, Pulkovskoye sh. 64, St.Petersburg, 196140, RUSSIA email: nila_lip@mail.ru

${ }^{2}$ Department of Investigations for Physics, University of Sonora, Rosales y Blvd. Transversal, col. Centro, Edif. 3-I. Hermosillo, Sonora, 83000, MEXICO email: jsaucedo@cajeme.cifus.uson.mx

${ }^{3}$ Main Astronomical Observatory, RAS, Pulkovskoye sh. St.Petersburg, 196140, RUSSIA email: km@gao.spb.ru

\begin{abstract}
In the present work the results of an optical spectroscopy study of 8 galaxies projected in the central part of the A1367 cluster are reported. The observations were made using the $2.2 \mathrm{~m}$. Guillermo Haro Telescope in Cananea (Mexico), and the Boller and Chivens spectrograph. A catalog of the optical positions of more than 100 galaxies in the cluster is presented. The redshifts of 7 galaxies were determined from their absorption systems, while for the remaining galaxy was obtained from its strong emission lines of H-alpha, S II, O III and H-beta. For one of the galaxies the emission redshift is $\mathrm{z}=0.015$, not showing absorptions lines at the cluster redshifts $(z=0.026)$, which argues that it is located between the observer and the A1367 cluster.
\end{abstract}

Keywords. Clusters, Galaxies, optical spectra

The cluster A1367, located at $Z=0.021-0.025$ has more than 100 members for more than half of which their redshifts are available. In this work we report the spectroscopic investigation of 8 members. The observation were carried out with $2.12 \mathrm{~m}$. Guillermo Haro Telescope in Cananea (Mexico), and the Boller and Chivens spectrograph. Spectra of 40 galaxies with magnitudes from $14 \mathrm{~m}$ to $18 \mathrm{~m}$ were measured. The pre-processing stage included all essential steps of CCD image handling: taking into account bias, dark current and flat-fielding, as well as filtering out matrix drawbacks and cosmic rays. The processing itself consisted of subtracting spectral lines of the night sky and wavelength calibration with the use of the built-in He-Ar standard source. For the A1367 cluster we identify 7 absorption systems and 3 objects show bright emission lines. Measured redshift in emission for two galaxies ( $Z$ is more than 0.7 ) argue that they are located behind the A1367 cluster. But for the galaxy number 4 in the table the redshift measured with emission lines is 0.0154 , and no absorptions at redshifts of A1367 were found.

\begin{tabular}{l|lll|lll|l|l|l|l}
$\mathrm{N}$ & $\mathrm{RA}(2000.0)$ & $\mathrm{DEC}(2000.0)$ & $\mathrm{R}(\mathrm{mag})$ & $\mathrm{B}(\mathrm{mag})$ & $Z_{e m}$ & $Z_{a b s}$ \\
\hline 1 & 11 & 43 & 44.41 & 19 & 44 & 42.3 & 14.58 & 16.73 & 0.7676 & 0.7676 \\
2 & 11 & 43 & 53.56 & 19 & 44 & 22.1 & 13.54 & 15.61 & & 0.0199 \\
3 & 11 & 43 & 59.56 & 19 & 46 & 44.2 & 11.12 & 13.14 & & 0.0175 \\
4 & 11 & 44 & 1.90 & 19 & 47 & 3.9 & 11.93 & 12.18 & 0.0154 & \\
5 & 11 & 44 & 3.03 & 19 & 44 & 25.1 & 14.02 & 16.20 & & 0.0216 \\
6 & 11 & 44 & 5.34 & 19 & 45 & 12.0 & 14.57 & 15.78 & & 0.0032 \\
7 & 11 & 44 & 7.66 & 19 & 44 & 15.4 & 10.94 & 13.32 & 0.7798 & 0.0261 \\
8 & 11 & 44 & 8.74 & 19 & 45 & 20.2 & 14.63 & 16.39 & & 0.0014
\end{tabular}

